\title{
The application of proton spectrometers at the SG-III facility for ICF implosion areal density diagnostics
}

\author{
Xing Zhang, Jianhua Zheng, Ji Yan, Zhenghua Yang, Ming Su, Yudong Pu, Pin Yang, Xufei Xie, Li Chen, \\ Ming Chen, Tianxuan Huang, Shao'en Jiang, Shenye Liu, and Jiamin Yang \\ Laser Fusion Research Center, Chinese Academy of Engineering Physics, Mianyang, Sichuan 621900, China \\ (Received 19 May 2015; revised 7 September 2015; accepted 30 September 2015)
}

\begin{abstract}
Charged particle diagnostics is one of the required techniques for implosion areal density diagnostics at the SG-III facility. Several proton spectrometers are under development, and some preliminary areal density diagnostics have been carried out. The response of the key detector, CR39, to charged particles was investigated in detail. A new track profile simulation code based on a semi-empirical model was developed. The energy response of the CR39 detector was calibrated with the accelerator protons and alphas from a ${ }^{241} \mathrm{Am}$ source. A proton spectrometer based on the filtered CR39 detector was developed, and D-D primary proton measurements were implemented. A step range filter spectrometer was developed, and preliminary areal density diagnostics was carried out. A wedged range filter spectrometer array made of Si with a higher resolution was designed and developed at the SG-III facility. A particle response simulation code by the Monte Carlo method and a spectra unfolding code were developed. The capability was evaluated in detail by simulations.
\end{abstract}

Keywords: areal density diagnostics; CR39 detector; SRF proton spectrometer; WRF proton spectrometer

\section{Introduction}

The SG-III facility has 48 laser beams with a maximum output energy of $180 \mathrm{~kJ}$. It is a new platform for inertial confinement fusion (ICF) experiments. A high enough areal density $(\rho \mathbf{R})$ is necessary for ignition, and how to realize this is one of the most important issues in implosion physics research. Measurement of the energy downshift of the fusion protons is an effective technique in passive $\rho \mathrm{R}$ diagnostics. In $\mathrm{D}-\mathrm{D}$ fuel implosion, protons are emitted in a primary D-D reaction and a secondary D- ${ }^{3} \mathrm{He}$ reaction with energies of 3 and $12-17 \mathrm{MeV}$, respectively. In D- ${ }^{3} \mathrm{He}$ fuel implosion, protons from primary D- ${ }^{3} \mathrm{He}$, D-D and ${ }^{3} \mathrm{He}-{ }^{3} \mathrm{He}$ reactions are dominant. When the protons, generated in the core, pass through the fuel and shell plasmas, there will be an energy loss, which is related to $\rho \mathbf{R}$, due to the interaction with the plasma. The stopping power of the protons in dense plasma, quite different from that in non-ionized matter, can be calculated with some well-defined models, such as the Li-Petrasso (L-P) model $^{[1]}$ and the Brown-Preston-Singleton (BPS) model $^{[2]}$. This technique has been widely used in $\rho \mathrm{R}$ diagnostics at $\mathrm{NIF}^{[3]}$, OMEGA ${ }^{[4]}$, GEKKO-XII ${ }^{[5]}$ and some other ICF facilities. Moreover, the $\rho \mathbf{R}$ evolution in

Correspondence to: S. Liu, PO Box 919-986, No. 64 Mianshan Road, Mianyang, Sichuan province 621900, China. Email: 1sye1029@163.com
D- ${ }^{3} \mathrm{He}$ implosion can be diagnosed by the different energy downshifts at the shock bang time and compression bang time $^{[6,7]}$. Besides, the fuel areal density in D-D implosion can be diagnosed by the yield ratio of secondary protons and primary neutrons, and the residual $\mathrm{CH}$ ablator areal density in $\mathrm{D}-\mathrm{T}$ implosion can be diagnosed by the neutron recoil proton spectra. Through $\rho \mathrm{R}$ measurements at multiple view angles by several proton spectrometers, the compression asymmetry can be diagnosed ${ }^{[4]}$. Furthermore, the mix effect can be studied by fusion proton measurement in some special targets ${ }^{[7-10]}$. The measured ${ }^{3} \mathrm{He}-{ }^{3} \mathrm{He}$ proton spectra provide a new approach for laboratory astrophysics research.

Several kinds of proton spectrometer have been developed at NIF, OMEGA, NOVA, GEKKO-XII and some other ICF facilities, such as the step range filter (SRF) spectrometer ${ }^{[11,12]}$, the wedged range filter (WRF) spectrometer $^{[11,13]}$ and the magnet-based spectrometer ${ }^{[5,11]}$. The first two are compact spectrometers with high detection efficiencies and perform well in $\mathrm{D}-{ }^{3} \mathrm{He}$ reaction proton measurement. The last one has the highest energy resolution, while the space occupation and the cost are higher. For all of the spectrometers, the CR39 detector is the key detector because of the high sensitivity to proton, alpha and some other ionizing particles and quite low sensitivity to electron, x-ray and $\gamma$-ray. In this work, the development of the proton spectrometers for $\rho \mathrm{R}$ diagnostics at the 
SG-III facility is introduced. The detailed charged particle response of the CR39 detector is studied in Section 2. A new track profile simulation code is presented, as well as an experimental study of the energy response. A proton spectrometer based on the filtered CR39 detector and the DD primary proton measurements are described in Section 3. Section 4 describes the setup of a SRF spectrometer and preliminary $\rho \mathrm{R}$ diagnostics. Section 5 describes the setup of a Si-WRF spectrometer array at the SG-III facility and its capability evaluation. Some concluding remarks are listed in Section 6.

\section{Energy response of the CR39 detector to charged particles}

\subsection{Processing of the the CR39 detector}

The CR39 is a kind of solid-state nuclear track detector. It is a transparent plastic with a chemical composition of $\mathrm{C}_{12} \mathrm{H}_{18} \mathrm{O}_{7}$. When a charged particle passes through a CR39 detector, it deposits energy along the trail, mostly by ionization. A narrow damaged trail in the CR39, on the scale of $\mathrm{nm}$, is formed. The amount of damage along the trail is mainly determined by the local electronic stopping power of the incident particle ${ }^{[14]}$. When the irradiated CR39 is exposed in an etchant, the surface is etched at a bulk velocity $v_{\mathrm{B}}$, while the damaged trail is etched at a track velocity $v_{\mathrm{T}}$. A visible track, on the scale of $\mu \mathrm{m}$, is formed after etching. The appearance parameters of the tracks are related to the species and energy of the incident particles. The CR39 detector has been widely used in many fields, such as ICF proton diagnostics, dosimetry for dose assessment and particle detection in space ${ }^{[11,15-17]}$.

In this work, the etchant is $6 \mathrm{~mol} / \mathrm{L} \mathrm{NaOH}$ solution at a temperature of $80^{\circ} \mathrm{C}$. The typical etching time is $6 \mathrm{~h}$, and it can be set in a range of $2-9 \mathrm{~h}$ to make sure that the tracks are visible and there is no saturation. After etching, the CR39 detectors are rinsed with dilute acetic acid and highly purified water, and then dried. The appearance parameters of the particle track profiles are obtained from photos of the etched detector's surfaces, by a digital microscope with objective magnifications of $20 \times$ and $50 \times$. The spatial resolution of the track diameter is approximately 1 and $0.4 \mu \mathrm{m}$ for the two magnifications, respectively.

\subsection{Simulation of the track profile induced by a charged particle}

Three track parameters are mostly used in the particle analysis: diameter, contrast and eccentricity. The diameter is always used to deduce the particle energy and species, while the contrast and eccentricity are used to reject the intrinsic tracks and neutron induced tracks ${ }^{[11]}$. The track diameter has a nonlinear response to the particle energy, and it varies with the etching conditions. In the measurement with a specific etching condition (etchant, temperature and time), a welldefined empirical $V$-function ( $\left.V=v_{\mathrm{T}} / v_{\mathrm{B}}\right)$ based etching kinematics model can describe the response well, and it has been studied in many works ${ }^{[15,17,18]}$. In the application of ICF proton measurement, the etching time varies for different fusion yields and some other conditions. A semiempirical model ${ }^{[19]}$ of the $V$-function, independent of the etching time, has been proposed, as shown in Equation (1), where $\left(\frac{d E}{d x}\right)_{\text {elec }}$ is the local electronic stopping power along the trail, and $k$ and $n$ are the free parameters. In this section, a new etching kinematics simulation code based on a semi-empirical model is developed. The electronic stopping power is calculated by the TRIM code ${ }^{[20]}$. The track profiles of perpendicular incident particles and oblique incident particles are simulated.

$$
V=\frac{V_{\mathrm{T}}}{V_{\mathrm{B}}}=1+k\left(\frac{d E}{d x}\right)_{\text {elec }}^{n} .
$$

In the case of perpendicular incidence, the track profile can be calculated in two steps, as shown in Figure 1(a). The damaged trail is first etched to the point $A^{\prime}\left(y^{\prime}, z^{\prime}\right)$ in a time of $t^{\prime}$ at a velocity of $v_{\mathrm{T}}(z)$. Then, the wall of the nano-hole is etched at a velocity of $v_{\mathrm{B}}$ in the direction determined by the angle of $\xi$. In the time of $\left(t-t^{\prime}\right)$, the track profile reaches the point $A\left(y_{A}, z_{A}\right)$. The two-step process can be described by Equations (2)-(4). The bulk etching depth can be calculated by Equation (5). With Equations (2)-(5), the track profile is obtained, shown as the solid line in Figure 1(a). The diameter, $D$, of the track on the surface is calculated using Equation (6). If the etchant has reached the particle range position $\mathrm{R}$ during the etching time, while the bulk etching depth is still less than the range (overetched case), as shown in Figure 1(b), the etchant first etches the trail to the range position at $v_{\mathrm{T}}(z)$, and then etches around the wall at $v_{\mathrm{B}}$ to form a spherical bottom. The solution of the track profile is similar to that in the non-overetched case. If the bulk etching depth exceeds the range of the particle (fully overetched case), the track profile is spherical, and the track image will have a low contrast and will usually be rejected.

$$
\begin{gathered}
z^{\prime}=\int_{0}^{t^{\prime}} v_{\mathrm{T}}(z) d z, \\
\cos \xi=v_{\mathrm{B}} / v_{\mathrm{T}}, \\
z_{A}-z^{\prime}=v_{\mathrm{B}}\left(t-t^{\prime}\right) \cos \xi, \\
z_{A}=v_{\mathrm{B}} t, \\
D=2 y_{A}=2 v_{\mathrm{B}}\left(t-t^{\prime}\right) \sin \xi .
\end{gathered}
$$

In the case of oblique incidence, the track profile is solved by Equations (2)-(4) in $y^{\prime}-z^{\prime}$ coordinates and Equation (5) in $y-z$ coordinates, as shown in Figure 2. The shape of the track on the surface of the etched CR39 is no longer circular. 


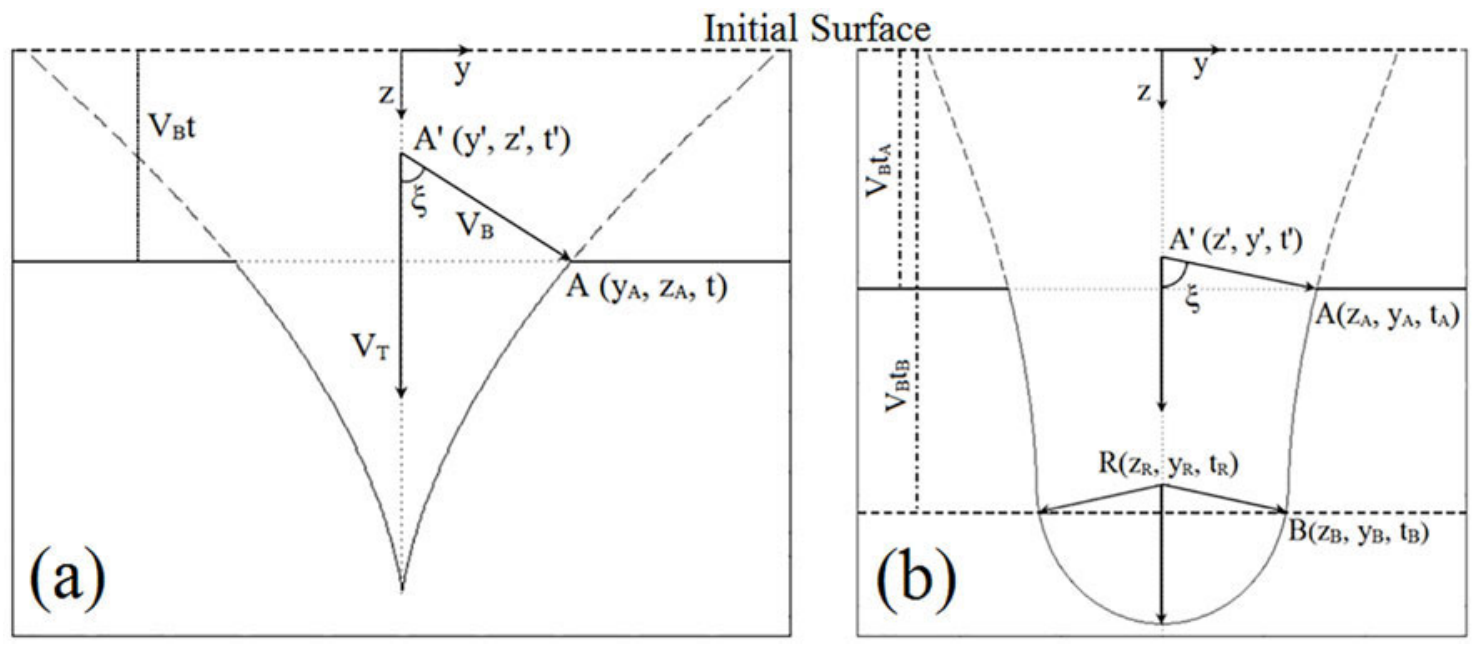

Figure 1. The track profiles in (a) the non-overetched case and (b) the overetched case for perpendicular incident particles.

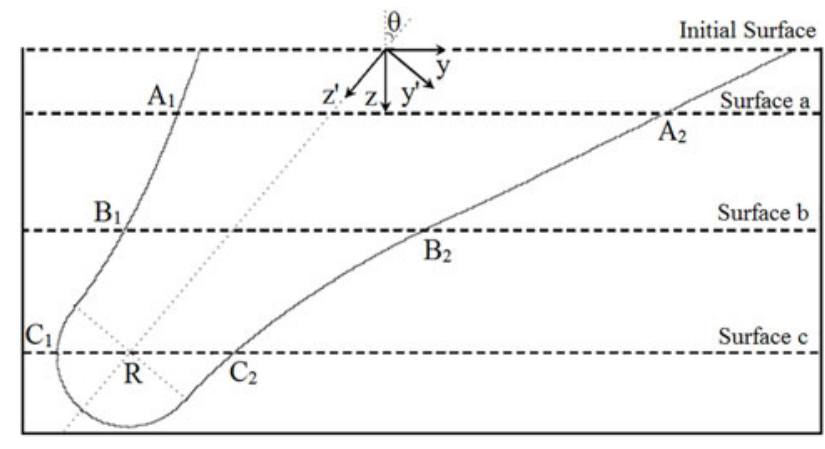

Figure 2. The track profiles for oblique incident particles.

If the bulk etching depth is not very large, for example surface $a$ or $b$, the surface track shape will be an ellipse. If the etching depth is very large, for example surface $c$, the shape will be an egg shape. This case often occurs in the tracks induced by neutron recoil protons.

\subsection{Energy response calibration}

The CR39 detector in this work was manufactured by Tastrak in UK. The energy responses of the track diameter to protons and alphas were measured at some energies, as the square and diamond points shown in Figure 3. For protons, the responses to 16 energies in the range of $0.7-$ $3 \mathrm{MeV}$ and three energies in the range of $4-10 \mathrm{MeV}$ were measured at a $2 \times 1.7 \mathrm{MV}$ tandem accelerator and a $2 \times 6 \mathrm{MV}$ tandem accelerator, respectively, at Peking University. The uncertainties in the proton energy were less than $1 \%$, and the incident proton beams were perpendicular to the CR39 surface. A timed aperture with a diameter of $1 \mathrm{~cm}$ was used to control the exposure time to avoid track saturation on the CR39. For alphas, the response to the $5.5 \mathrm{MeV}$ alpha particles from a ${ }^{241} \mathrm{Am}$ source was measured. To limit the

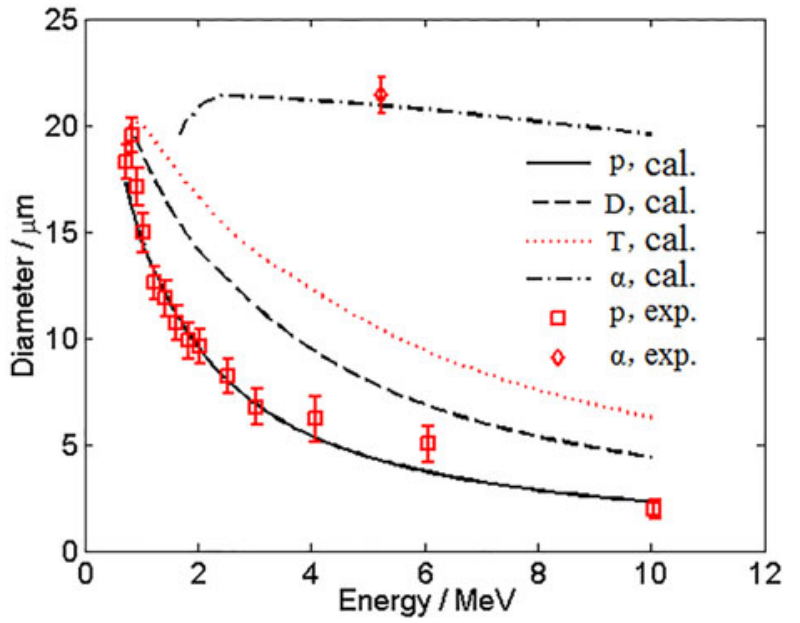

Figure 3. The measured and calculated energy responses of CR39 to proton, deuteron, triton and alpha.

incident angle and the number of the alpha particles, the timed aperture was placed in between, $20 \mathrm{~cm}$ away from the source and $10 \mathrm{~cm}$ away from the CR39 plastic. The area of the CR39 plastic used for track readout was approximately $1 \mathrm{~cm}^{2}$. The irradiated CR39 plastic was etched in $6 \mathrm{~mol} / \mathrm{L} \mathrm{NaOH}$ solution at a temperature of $80^{\circ} \mathrm{C}$ for $6 \mathrm{~h}$. By the mass loss of the unirradiated CR39 plastic before and after etching, the bulk etching velocity was measured as $\mathrm{v}_{\mathrm{B}}=1.793 \pm 0.015 \mu \mathrm{m} / \mathrm{h}$. By iterative fitting of the track diameters to the particle energies, the free parameters in Equation (1) were obtained as $k=3.3 \times 10^{-4}$ and $n=2.4$. The calculated energy responses of the diameter to proton, deuteron, triton and alpha are also shown in Figure 3. With increase of the particle energy, the diameter decreases and the diameter decrease rate also decreases. Considering the track image spatial resolution and requirement of particle energy resolution, in routine proton measurements at the SG-III facility, readout proton tracks with diameters just in 
the range of 5-15 $\mu \mathrm{m}$ are used. Reference [21] reported that the piece-to-piece variation of the proton track diameter was approximately 19\%. Considering the same etching conditions in this work as those in Ref. [21], in the following simulation work, a Gaussian distributed track diameter with the mean value of Figure 3 and a deviation of $19 \%$ was adopted. The detection efficiency was calculated as the probability that the track diameter would fall in the range of 5-15 $\mu \mathrm{m}$.

\section{D-D fusion primary proton measurement by a filtered CR39 detector}

In low yield $\mathrm{D}_{2}$ fuel implosion experiments, the energy downshift of the primary proton spectra is a possible way to diagnose the areal density, which is less than $30 \mathrm{mg} / \mathrm{cm}^{2}$. The mean energy of the protons emitted in the hot-spot region is $3 \mathrm{MeV}$, with a Doppler broadening related to the ion temperature. When the areal density is less than $10 \mathrm{mg} / \mathrm{cm}^{2}$, the energy downshift is approximately linear with the areal density. In laser directly driven implosion, the disturbance comes from the ablator protons and the radial acceleration effect. Most ablator protons have low energy $(<1 \mathrm{MeV})$ and can be shielded with an appropriate filter. When the proton emission time falls in the laser pulse duration, the radial electric field in the corona region will accelerate the protons and contribute an energy upshift to the measured spectra, which will present a lower diagnosed $\rho$ R. Distinct energy upshifts for different experimental conditions are necessary. However, the relatively different proton energy downshifts can be used to infer the variation of $\rho \mathrm{R}$ in similar shots. The typical energy upshift of $\mathrm{D}-\mathrm{D}$ reaction protons at OMEGA is approximately $0.5 \mathrm{MeV}^{[22]}$.

In laser directly driven cool gas target and cryogenic $\mathrm{D}_{2}$ target implosion experiments at the SG-III prototype facility, the D-D fusion primary proton spectra were also measured.
The cool gas target has an inner radius of $381 \mu \mathrm{m}$ and a $\mathrm{CH}$ shell thickness of $9 \mu \mathrm{m}$, filled with $\mathrm{D}_{2}$ gas $(30 \mathrm{~K}, 200 \mathrm{kPa})$. The cryogenic target has an inner radius of $389 \mu \mathrm{m}$, a $\mathrm{CH}$ shell thickness of $9 \mu \mathrm{m}$ and an inner $D_{2}$ ice thickness of $40 \mu \mathrm{m}$. There were eight laser beams (1 ns pulse duration and $6.4 \mathrm{~kJ}$ total energy) driving the targets symmetrically. Due to the low fusion yield $\left(\sim 10^{7}\right)$, an Al filtered CR39 detector was adopted to increase the detection efficiency. The schematic experimental setup is shown in Figure 4(a). The detector was set up below the cryogenic shielding, facing the laser entrance hole (LEH). The distance between the target and the CR39 was approximately $25 \mathrm{~cm}$. Al filters with thicknesses of 20 and $25 \mu \mathrm{m}$ were used for the cool gas target and the cryogenic target, respectively. The CR39 recorded proton spectra were obtained based on the proton track histograms. With the TRIM calculated Al filter induced proton energy loss matrices in the energy range of $1-5 \mathrm{MeV}$, the target emission proton spectra were obtained as shown in Figure 4(b). It can be seen that the proton energy downshift of the cryogenic target was higher than that of the cool gas target, which represented a higher implosion areal density. Some further study of the energy upshift due to radial electric field acceleration will be carried out, to give the proton energy downshift in the fuel and shell and quantify the areal density.

\section{D- ${ }^{3}$ He reaction proton measurement by SRF spec- trometer}

\subsection{Design and response simulation of the SRF spectrometer}

In ICF implosions, D- ${ }^{3} \mathrm{He}$ reaction protons are emitted by the primary reaction in the D- ${ }^{3} \mathrm{He}$ fuel implosion and the secondary reaction in the D-D fuel implosion. The proton yield and the energy downshift can be used to deduce
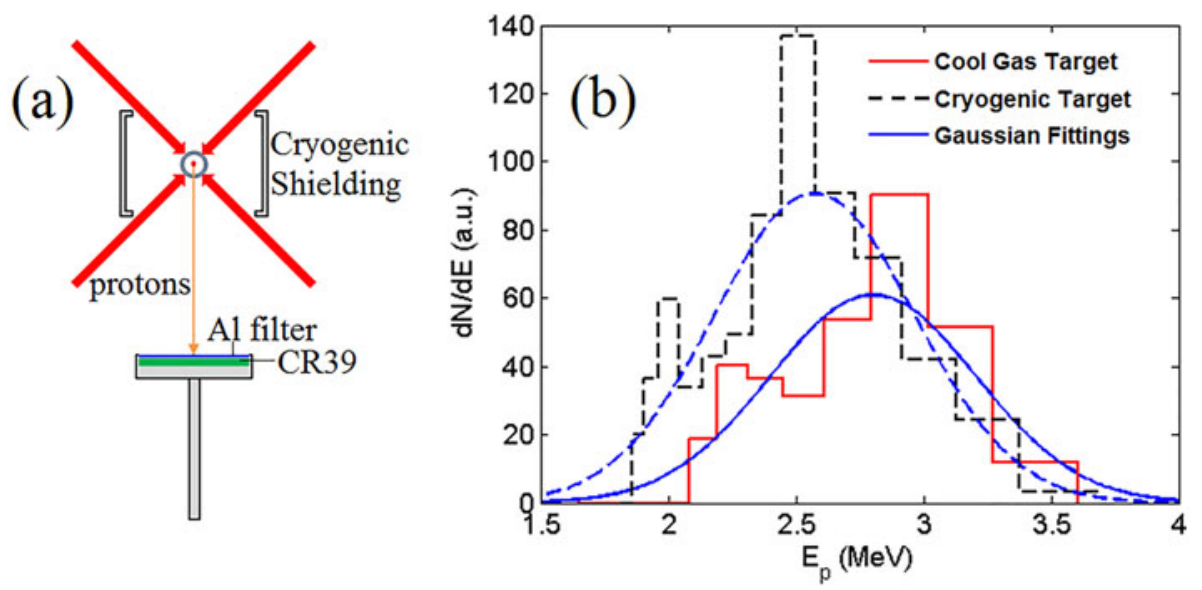

Figure 4. (a) The schematic setup of the D-D primary proton spectrometer at the SG-III prototype facility and (b) the measured proton spectra in the cool gas target (solid histogram) and the cryogenic $\mathrm{D}_{2}$ target (dashed histogram) experiments with Gaussian fitting lines (blue lines). 

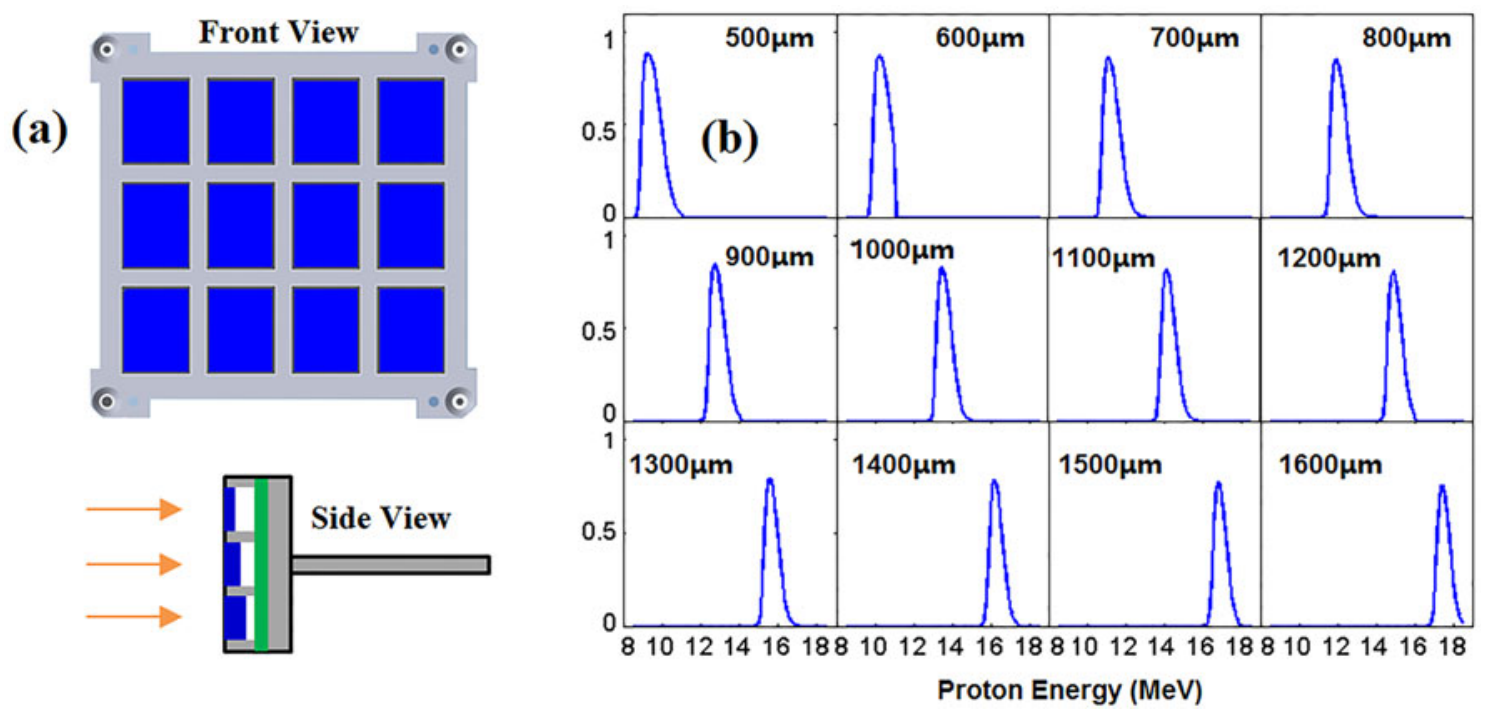

Figure 5. (a) The schematic diagram of the SRF proton spectrometer and (b) the simulated proton energy response matrix.

the areal density ${ }^{[4,19]}$. The high average proton energy $(15 \mathrm{MeV})$ makes it possible to diagnose the areal density up to $100 \mathrm{mg} / \mathrm{cm}^{2}$. As a result, the energy dynamic range of the spectrometer is required to be wide, $8-18 \mathrm{MeV}$ for example.

The SRF proton spectrometer uses a range of stepped filters to measure protons in different energy ranges ${ }^{[12]}$. An SRF proton spectrometer has been built at the SG-III facility for $\mathrm{D}^{3} \mathrm{He}$ reaction proton measurement. The schematic diagram is shown in Figure 5(a). A set of 12 Al filters, with thicknesses from 500 to $1800 \mu \mathrm{m}$ with an interval of $100 \mu \mathrm{m}$, was adopted in the SRF spectrometer. The CR39 detector was divided into 12 parts to record the transmitted protons. Proton tracks with diameters in the range of 5$15 \mu \mathrm{m}$ were used. The low energy protons were ranged out by the filter to each cell, while the high energy protons were not counted by the CR39. Every cell in the spectrometer looked like an energy band-pass filter to the protons. In the response function simulation, the track diameters of the transmitted protons were calculated according to a Gaussian distribution with the mean values of Figure 3 and a deviation of $19 \%$. The response function of each cell to proton energy is shown in Figure 5(b). With the help of the energy response matrix, a spectrum unfolding code based on a weighted averaging algorithm was also developed.

\subsection{D-D implosion secondary proton measurement on the $S G$-III facility}

The SRF proton spectrometer was first used in a $\mathrm{D}_{2}$ exploding pusher target experiment on the SG-III facility in 2014. The spectrometer was installed on a general diagnostics instrument manipulator (DIM) and accessed the chamber in the middle plane. The distance between the target and the spectrometer was approximately $16 \mathrm{~cm}$. The

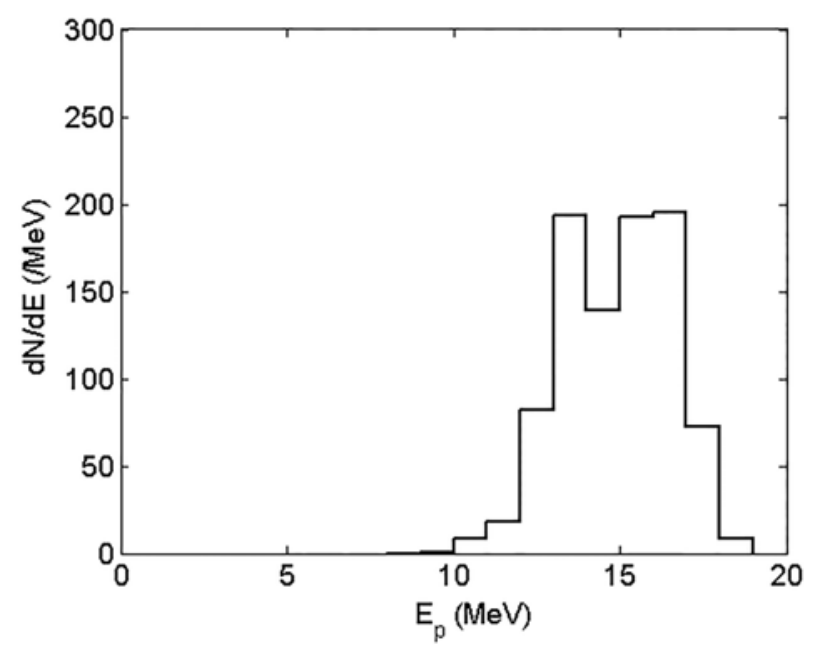

Figure 6. The measured secondary proton spectra in the shot with a target diameter of $800 \mu \mathrm{m}$.

D-D secondary proton spectra in three shots were measured. The three targets had the same shell of $2 \mu \mathrm{m}$ thick $\mathrm{SiO}_{2}$, while they had different diameters of 800,620 and $450 \mu \mathrm{m}$. They were filled with $10 \mathrm{~atm} \mathrm{D}_{2}$ fuel. There were 32 laser beams directly driving the target, with a total energy of $30 \mathrm{~kJ}$. In each shot, the hit positions on the target surface of the laser beams were shifted a little to decrease the root mean square (RMS) of the laser illumination. The measured proton spectrum for the $800 \mu \mathrm{m}$ diameter target is shown in Figure 6. The spectra in the other two shots were similar. The emitted protons were distributed mainly in the energy range of 12-18 MeV. There were no significant energy downshifts. This was consistent with expectations. Because the areal density of the exploding pusher target was very low, expected to be less than $1 \mathrm{mg} / \mathrm{cm}^{2}$, the energy downshift of secondary 
protons would be approximately $0.1 \mathrm{MeV}$, which was too small compared with the spectrometer energy resolution. However, the results show that the SRF spectrometer works well and is able to diagnose the areal density when it is higher than $10 \mathrm{mg} / \mathrm{cm}^{2}$. Moreover, from the spectra, the yields of the secondary protons were measured as $4.0 \times 10^{6}$, $1.9 \times 10^{6}$ and $9 \times 10^{5}$, respectively. The neutron yields in the three shots were $3.1 \times 10^{6}, 1.9 \times 10^{6}$ and $1.7 \times 10^{6}$, respectively. A Monte Carlo code was developed to calculate the secondary proton emission, including the uniform hotspot model and the ${ }^{3} \mathrm{He}^{+}$stopping power data calculated by the L-P model. According to the measured yield ratios of the secondary proton to primary neutron ${ }^{[23]}$, the estimated areal densities of the three shots were $0.8,0.7$ and $0.4 \mathrm{mg} / \mathrm{cm}^{2}$, respectively.

\section{A Si-WRF proton spectrometer for high energy pro- ton diagnostics}

\subsection{Design and response simulation of the $\mathrm{Si}-W R F$ spectrometer}

To overcome the disadvantage of low energy resolution in the SRF spectrometer, the WRF spectrometer was developed, which uses a wedged filter to replace the filter package. As illustrated in Figure 7(b), for incident mono-energetic protons, in the thicker end of the filter the protons will be ranged out, while in the thinner end the tracks of the transmitted protons will not be counted because of a track diameter smaller than $5 \mu \mathrm{m}$. The readout proton tracks are distributed in a narrow band on the surface of the CR39. By unfolding the proton track position spectra with the help of a well-defined energy response matrix, a high resolution incident proton spectrum can be obtained. There are already four types of WRF spectrometer, made from $\mathrm{Al}$ or $\mathrm{ZrO}_{2}$ filters, developed at the OMEGA and NIF facilities ${ }^{[13]}$. A WRF spectrometer, made from a Si filter, has been set up at the SG-III facility for 6-18 MeV proton measurement. The Si filter has similar characteristics to the Al filter in the particle range, but the deformation is much smaller during storage. The structure of a single spectrometer is illustrated in Figure 7(b). A WRF spectrometer array consisting of five spectrometers was developed, as shown in Figure 7(a). The thickness range of the Si filter is $120-2000 \mu \mathrm{m}$. Both the width and length of the filter are $4 \mathrm{~mm}$. The WRF array will be installed on a DIM and will access the chamber in the middle plane. There will be three view angles in the poloidal direction and three in the azimuthal direction, which may be useful in implosion asymmetry diagnostics. The distance between the target and the array can be adjusted in the range of $16-50 \mathrm{~cm}$. The maximum poloidal and azimuthal view angles are $\pm 20^{\circ}$.

A Monte Carlo simulation code based on the Geant4 package ${ }^{[24]}$ was developed to study the spectrometer response and evaluate its capability. The simulated proton
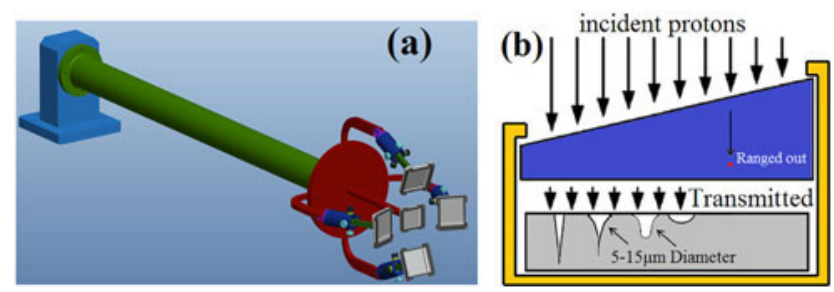

Figure 7. (a) The alignment of the Si-WRF spectrometer array at the SG-III facility (b) and a schematic illustration of a single WRF spectrometer ${ }^{[1]}$.

position spectra for 9 and $15 \mathrm{MeV}$ mono-energetic protons are shown in Figure $8(\mathrm{a})$. The $1-4 \mathrm{MeV}$ transmitted proton tracks are distributed in a $3 \mathrm{~mm}$ wide band. This indicates that the bin width of the recorded proton position spectra should be no more than $1 \mathrm{~mm}$. The proton energy responses in the range of 4-19 MeV were also simulated with an energy interval of $0.1 \mathrm{MeV}$, as shown in Figure 8(b). This shows that the designed WRF spectrometer is able to work well in the range of 6-18 MeV. It can also be used as a well-defined energy response matrix in the following spectra unfolding code.

\subsection{Capability evaluation}

First, the capability of energy spectra reconstruction was evaluated. The test 'experimental' proton position spectra were simulated in the cases of different incident proton energy distributions. The spectra were unfolded by the MAXED code ${ }^{[25]}$, which uses the maximum entropy algorithm. The energy response matrix was the simulated one in Figure 8(b). In the cases of mono-energetic proton incidence, the broadenings of the unfolded energy spectra were approximately $0.2 \mathrm{MeV}$, which represented the energy resolution of the WRF spectrometer. In the case of D- ${ }^{3} \mathrm{He}$ implosion primary proton incidence, two input Gaussian spectra were adopted with mean energies of 15 and $12 \mathrm{MeV}$ and broadenings of 405 and $780 \mathrm{keV}$, respectively, as shown in Figure 9(a). The one with higher energy represented the proton emission spectrum at $5 \mathrm{keV}$ ion temperature without energy downshift, while the other one represented the energy-downshifted proton spectrum in an areal density of $60-70 \mathrm{mg} / \mathrm{cm}^{2}$. In the simulated 'experiment' with $10^{6}$ proton incidence, the unfolded spectra are also shown in Figure 9(a). They are consistent with the input Gaussian spectra. In the case of D-D implosion secondary proton incidence, a rectangular spectrum of $12-17 \mathrm{MeV}$ was adopted as the simulation input. The unfolded spectrum and the input spectrum are consistent, as shown in Figure 9(b).

Second, the effect of the proton counts was investigated. In the case of $\mathrm{D}-{ }^{3} \mathrm{He}$ implosion, with an incident proton count of $10^{5}-10^{8}$, which represents a yield range of $10^{8}-$ $10^{11}$, the unfolded spectra are shown in Figure 10(a). This shows that when the D- ${ }^{3} \mathrm{He}$ primary proton yield exceeds 

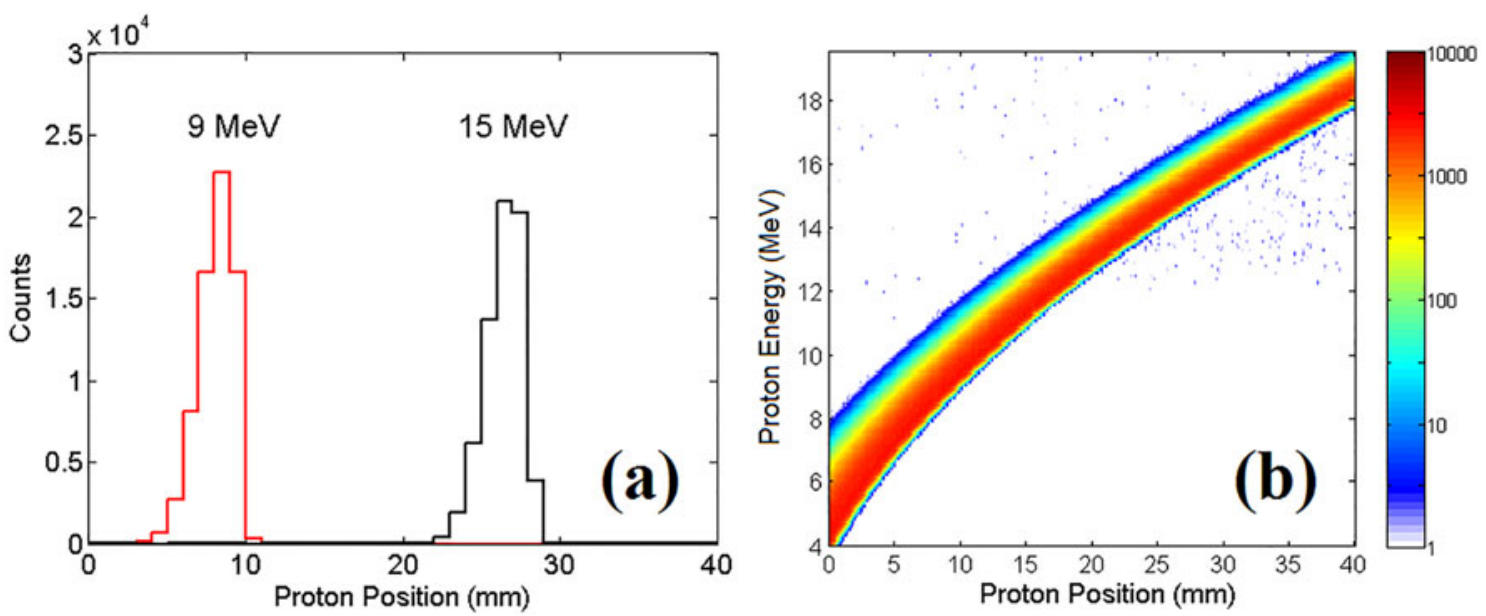

Figure 8. (a) The energy response to 9 and $15 \mathrm{MeV}$ mono-energetic protons and (b) the energy response matrix in the range of 4-19 MeV.
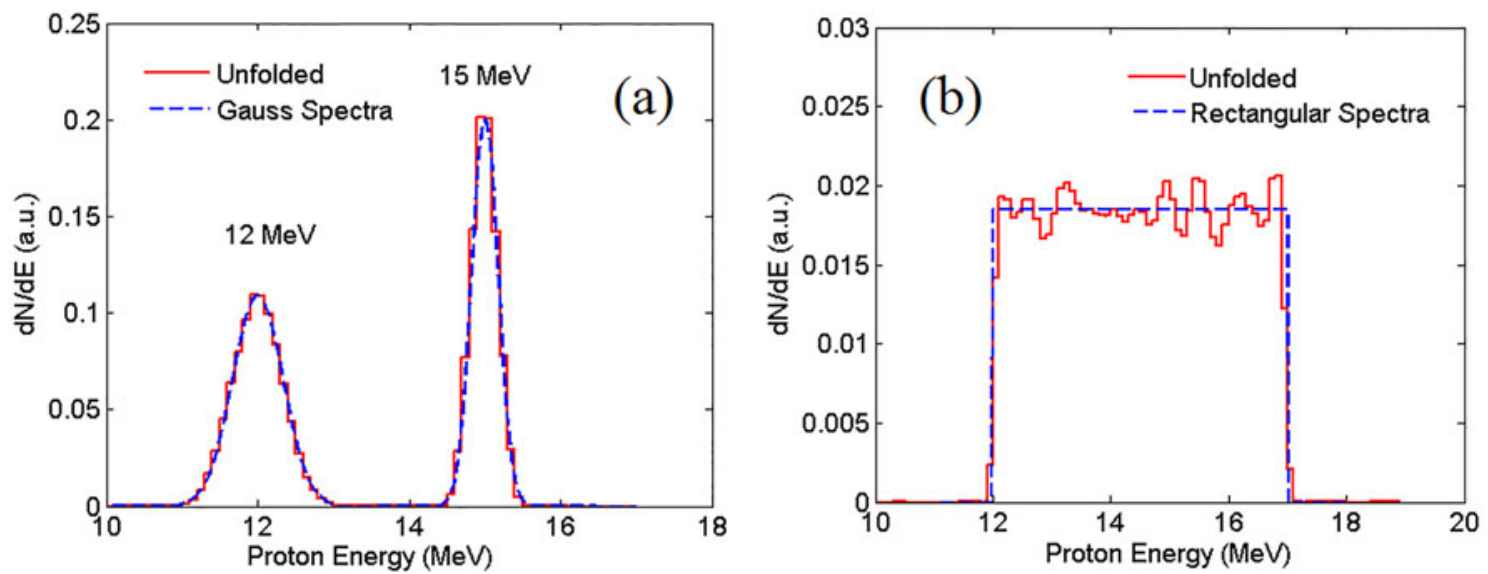

Figure 9. (a) Comparisons of the unfolded spectra and the simulation input spectra in the cases of D- ${ }^{3} \mathrm{He}$ primary proton and (b) D-D secondary proton.
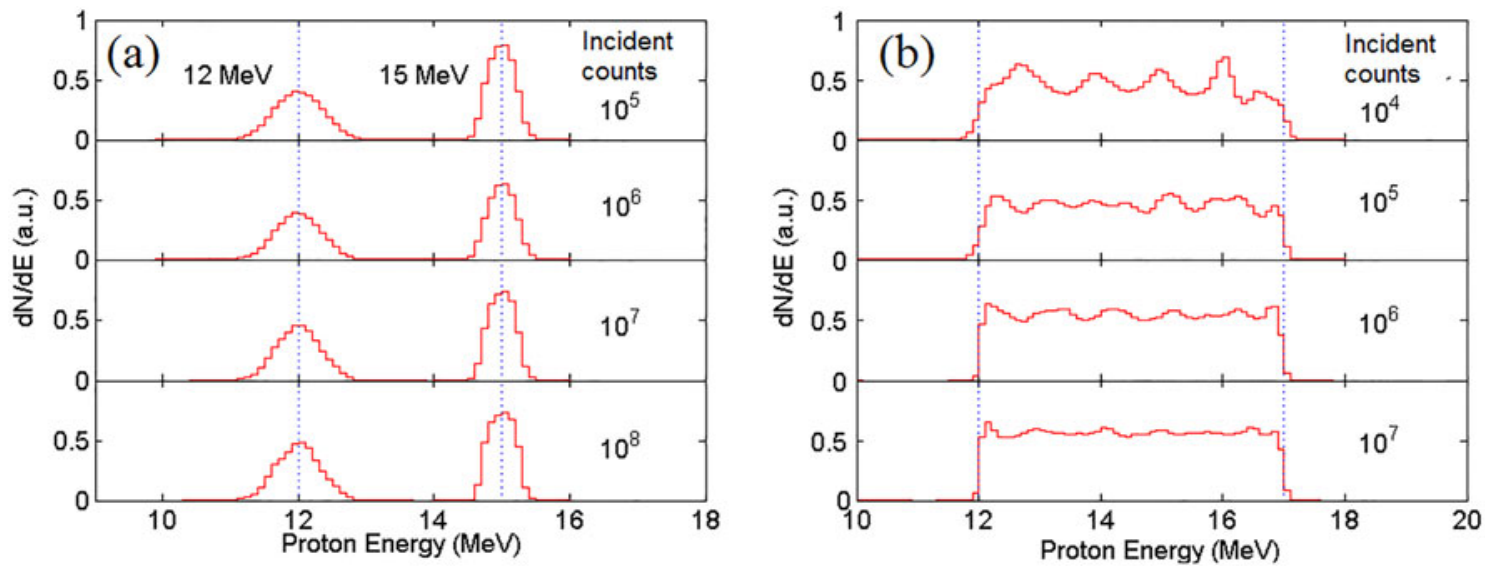

Figure 10. The unfolded energy spectra under different incident proton counts in the cases of (a) D- ${ }^{3}$ He primary protons and (b) D-D secondary protons .

$10^{8}$, the WRF spectrometer can work well. In the case of D-D implosion, with an incident secondary count of $10^{4}$ $10^{7}$, which represents a primary yield range of $10^{10}-10^{13}$, the unfolded spectra are shown in Figure 10(b). This shows that the necessary yield for WRF spectrometer measurement is approximately $10^{10}$. 


\section{Conclusions}

In this work, three kinds of CR39-based proton spectrometers were applied at the SG-III facility for ICF implosion areal density diagnostics. The energy response of the CR39 detector to charged particles was studied by simulations and experiments. A Geant 4 particle transport code including the track profile calculation code will be developed to study the background track profiles of the scattered particles and neutron recoil protons. A filtered CR39 detector has been applied in the measurement of D-D primary protons in low yield shots. A preliminary analysis of the areal density variation was implemented at the SG-III prototype facility. For the yields and spectra measurements of the D- ${ }^{3} \mathrm{He}$ reaction protons at the SG-III facility, a SRF spectrometer has already been applied in D-D fuel implosion areal density diagnostics. A Si-WRF spectrometer array has been designed and developed at the SG-III facility. Its capability was evaluated by Geant 4 simulations in detail. In the following D ${ }^{3} \mathrm{He}$ fuel implosion experiments, it will be applied in the diagnostics of areal density and asymmetry, mix effect studies and some plasma nuclear physics studies. By installing a $\mathrm{CH}$ foil in front, it can be upgraded to a compact nWRF spectrometer ${ }^{[26]}$ for D-T reaction neutron spectra measurement. Moreover, for high resolution measurement of the charged particle spectra, a magnet-based charged particle spectrometer is also under development at the SG-III facility.

\section{Acknowledgements}

This work was supported by the Open Project of the State Key Laboratory of Nuclear Physics and Technology at Peking University (No. 2014-02). The authors are very grateful to Professor Xiaotang Ren and Professor Hongji Ma in Peking University for the operation of the two accelerators. The authors are also very grateful to Professor Tieshuan Fan and his team in Peking University for their help with the spectra unfolding code.

\section{References}

1. C. K. Li and R. D. Petrasso, Phys. Rev. Lett. 70, 3059 (1993).

2. L. S. Browna, D. L. Preston, and R. L. Singleton, Jr., Phys. Rep. 410, 237 (2005).

3. A. B. Zylstra, J. A. Frenje, F. H. Séguin, M. J. Rosenberg, H. G. Rinderknecht, M. Gatu Johnson, D. T. Casey, N. Sinenian, M. J.-E. Manuel, C. J. Waugh, H. W. Sio, C. K. Li, R. D. Petrasso, S. Friedrich, K. Knittel, R. Bionta, M. McKernan, D. Callahan, G. W. Collins, E. Dewald, T. Döppner, M. J. Edwards, S. Glenzer, D. G. Hicks, O. L. Landen, R. London, A. Mackinnon, N. Meezan, R. R. Prasad, J. Ralph, M. Richardson, J. R. Rygg, S. Sepke, S. Weber, R. Zacharias, E. Moses, J. Kilkenny, A. Nikroo, T. C. Sangster, V. Glebov, C. Stoeckl, R. Olson, R. J. Leeper, J. Kline, G. Kyrala, and D. Wilson, Rev. Sci. Instrum. 83, 10D901 (2012).
4. F. H. Séguin, C. K. Li, J. A. Frenje, D. G. Hicks, K. M. Green, S. Kurebayashi, R. D. Petrasso, J. M. Soures, D. D. Meyerhofer, V. Yu. Glebov, P. B. Radha, C. Stoeckl, S. Roberts, C. Sorce, T. C. Sangster, M. D. Cable, K. Fletcher, and S. Padalino, Phys. Plasmas 9, 2725 (2002).

5. Y. Kitagawa, K. A. Tanaka, M. Nakai, T. Yamanaka, K. Nishihara, H. Azechi, N. Miyanaga, T. Norimatsu, T. Kanabe, C. Chen, A. Richard, M. Sato, H. Furukawa, and S. Nakai, Phys. Rev. Lett. 75, 3130 (1995).

6. J. A. Frenje, C. K. Li, F. H. Séguin, J. Deciantis, S. Kurebayashi, J. R. Rygg, R. D. Petrasso, J. Delettrez, V. Yu. Glebov, C. Stoeckl, F. J. Marshall, D. D. Meyerhofer, T. C. Sangster, V. A. Smalyuk, and J. M. Soures, Phys. Plasmas 11, 2798 (2004).

7. C. K. Li, F. H. Séguin, J. A. Frenje, M. Rosenberg, R. D. Petrasso, P. A. Amendt, J. A. Koch, O. L. Landen, H. S. Park, H. F. Robey, R. P. J. Town, A. Casner, F. Philippe, R. Betti, J. P. Knauer, D. D. Meyerhofer, C. A. Back, J. D. Kilkenny, and A. Nikroo, Science 327, 1231 (2010).

8. J. R. Rygg, J. A. Frenje, C. K. Li, F. H. Séguin, R. D. Petrasso, V. Y. Glebov, D. D. Meyerhofer, T. C. Sangster, and C. Stoeckl, Phys. Rev. Lett. 98, 215002 (2007).

9. H. G. Rinderknecht, H. Sio, C. K. Li, A. B. Zylstra, M. J. Rosenberg, P. Amendt, J. Delettrez, C. Bellei, J. A. Frenje, M. Gatu Johnson, F. H. Séguin, R. D. Petrasso, R. Betti, V. Yu. Glebov, D. D. Meyerhofer, T. C. Sangster, C. Stoeckl, O. Landen, V. A. Smalyuk, S. Wilks, A. Greenwood, and A. Nikroo, Phys. Rev. Lett. 112, 135001 (2014).

10. D. T. Casey, D. T. Woods, V. A. Smalyuk, O. A. Hurricane, V. Y. Glebov, C. Stoeckl, W. Theobald, R. Wallace, A. Nikroo, M. Schoff, K. J. Wu, J. A. Frenje, O. L. Landen, B. A. Remington, and G. Glendinning, Phys. Rev. Lett. 114, 205002 (2015)

11. F. H. Séguin, J. A. Frenje, C. K. Li, D. G. Hicks, S. Kurebayashi, J. R. Rygg, B.-E. Schwartz, R. D. Petrasso, S. Roberts, J. M. Soures, D. D. Meyerhofer, T. C. Sangster, J. P. Knauer, C. Sorce, V. Yu. Glebov, C. Stoeckl, T. W. Phillips, R. J. Leeper, K. Fletcher, and S. Padalino, Rev. Sci. Instrum. 74, 975 (2003).

12. M. J. Rosenberg, A. B. Zylstra, J. A. Frenje, H. G. Rinderknecht, M. Gatu Johnson, C. J. Waugh, F. H. Séguin, H. Sio, N. Sinenian, C. K. Li, R. D. Petrasso, V. Yu. Glebov, M. Hohenberger, C. Stoeckl, T. C. Sangster, C. B. Yeamans, S. LePape, A. J. Mackinnon, R. M. Bionta, B. Talison, D. T. Casey, O. L. Landen, M. J. Moran, R. A. Zacharias, J. D. Kilkenny, and A. Nikroo, Rev. Sci. Instrum. 85, 103504 (2014).

13. F. H. Séguin, N. Sinenian, M. Rosenberg, A. Zylstra, M. J.E. Manuel, H. Sio, C. Waugh, H. G. Rinderknecht, M. Gatu Johnson, J. Frenje, C. K. Li, R. Petrasso, T. C. Sangster, and S. Roberts, Rev. Sci. Instrum. 83, 10 D908 (2012).

14. R. L. Fleischer, P. B. Price, and R. M. Walker, J. Appl. Phys. 36, 3645 (1965).

15. M. Caresana, M. Ferrarini, M. Fuerstner, and S. Mayer, Nucl. Instrum. Methods Phys. Res. A 683, 8 (2012).

16. G. Immè, D. Morelli, M. Aranzulla, R. Catalano, and G. Mangano, Radiat. Meas. 50, 253 (2013).

17. D. Zhou, E. Semones, S. Guetersloh, N. Zapp, M. Weyland, and E. R. Benton, Radiat. Meas. 45, 916 (2010).

18. D. Nikezic and K. N. Yu, Comput. Phys. Comm. 174, 160 (2006).

19. D. G. Hicks, "Charged-particle spectroscopy: a new window on inertial confinement fusion", PhD. Thesis (Massachusetts Institute of Technology, 1999). 
20. J. F. Ziegler, M. D. Ziegler, and J. P. Biersack, Nucl. Instrum. Methods Phys. Res. B 268, 1818 (2010).

21. N. Sinenian, M. J. Rosenberg, M. Manuel, S. C. McDuffee, D. T. Casey, A. B. Zylstra, H. G. Rinderknecht, M. Gatu Johnson, F. H. Séguin, J. A. Frenje, C. K. Li, and R. D. Petrasso, Rev. Sci. Instrum. 82, 103303 (2011).

22. D. G. Hicks, C. K. Li, F. H. Séguin, A. K. Ram, J. A. Frenje, R. D. Petrasso, J. M. Soures, V. Yu. Glebov, D. D. Meyerhofer, S. Roberts, C. Sorce, C. Stockl, T. C. Sangster, and T. W. Phillips, Phys. Plasmas 7, 5106 (2000).

23. M. D. Cable and S. P. Hatchett, J. Appl. Phys. 62, 2233 (1987).

24. S. Agostinelli, J. Allisonas, K. Amako, J. Apostolakis, H. Araujo, P. Arce, M. Asai, D. Axen, S. Banerjee, G. Barrand, F. Behner, L. Bellagamba, J. Boudreau, L. Broglia, A. Brunengo, H. Burkhardt, S. Chauvie, J. Chuma, R. Chytracek, G. Cooperman, G. Cosmo, P. Degtyarenko, A. DellAcqua, G. Depaola, D. Dietrich, R. Enami, A. Feliciello, C. Ferguson, H. Fesefeldt, G. Folger, F. Foppiano, A. Forti, S. Garelli, S. Giani, R. Giannitrapani, D. Gibin, J. J. G. Cadenas, I. Gonzalez, G. G. Abril, G. Greeniaus, W. Greiner, V. Grichine, A. Grossheim, S. Guatelli, P. Gumplinger, R. Hamatsu, K. Hashimoto, H. Hasui, A. Heikkinen, A. Howard, V. Ivanchenko, A. Johnson, F. W. Jones, J. Kallenbach, N. Kanaya, M. Kawabata, Y. Kawabata,
M. Kawaguti, S. Kelner, P. Kent, A. Kimura, T. Kodama, R. Kokoulin, M. Kossov, H. Kurashige, E. Lamanna, T. Lampen, V. Lara, V. Lefebure, F. Lei, M. Liendl, W. Lockman, F. Longo, S. Magni, M. Maire, E. Medernach, K. Minamimoto, P. M. Freitas, Y. Morita, K. Murakami, M. Nagamatu, R. Nartallo, P. Nieminen, T. Nishimura, K. Ohtsubo, M. Okamura, S. O'Neale, Y. Oohata, K. Paech, J. Perl, A. Pfeiffer, M. G. Pia, F. Ranjard, A. Rybin, S. Sadilov, E. Di Salvo, G. Santin, T. Sasaki, N. Savvas, Y. Sawada, S. Scherer, S. Sei, V. Sirotenko, D. Smith, N. Starkov, H. Stoecker, J. Sulkimo, M. Takahata, S. Tanaka, E. Tcherniaev, E. S. Tehrani, M. Tropeano, P. Truscott, H. Uno, L. Urban, P. Urban, M. Verderi, A. Walkden, W. Wander, H. Weber, J. P. Wellisch, T. Wenaus, D. C. Williams, D. Wright, T. Yamada, H. Yoshida, and D. Zschiesche, Nucl. Instrum. Methods Phys. Res. A 506, 250 (2003).

25. M. Reginatto, P. Goldhagen, and S. Neumann, Nucl. Instrum. Methods Phys. Res. A 476, 242 (2002).

26. A. B. Zylstra, M. Gatu Johnson, J. A. Frenje, F. H. Séguin, H. G. Rinderknecht, M. J. Rosenberg, H. W. Sio, C. K. Li, R. D. Petrasso, M. McCluskey, D. Mastrosimone, V. Y. Glebov, C. Forrest, C. Stoeckl, and T. C. Sangster, Rev. Sci. Instrum. 85, 063502 (2014). 